\title{
Fundamental solution of the Laplacian on flat tori and boundary value problems for the planar Poisson equation in rectangles
}

\section{Malik Mamode*}

\section{"Correspondence:}

malik.mamode@univ-reunion.fr Laboratoire PIMENT, Université de la Réunion, Saint-Denis, 97400,

Réunion, France

\begin{abstract}
The fundamental solution of the Laplacian on flat tori is obtained using Eisenstein's approach to elliptic functions via infinite series over lattices in the complex plane. Most boundary value problems stated for the planar Poisson equation in a rectangle for which series-only representations of solution were known, may thus be solved explicitly in closed-form using the method of images. Moreover, the fundamental solution of $n$-Laplacian on flat tori may also be simply derived by a convolution power.
\end{abstract}

PACS Codes: 02.30 .Em; 02.30.Jr

Keywords: 2D Poisson equation; Green function; horizon; boundary value problem; flat torus; analytical solution

\section{Introduction}

The Poisson equation is certainly the simplest and the most famous partial differential equation of elliptic type which arises in many areas of mathematical physics, for example for steady state problems in electrostatics, heat conduction and fluid flow. The studies of boundary value problems (BVPs) for Poisson equation in finite or semi-infinite connected domains are ancient and well documented in many reference books [1-3]. Different analytical methods to construct solutions of these problems exist such as separation of variables, eigenfunction expansion, etc. and may depend on the nature of boundary conditions and the shape of domains. Among these methods, the method of source function, also called the Green function method, is the only one giving convenient and compact analytical representation of solutions in two- or three-dimensional space. In this method, the BVPs are reformulated into integral equations that involve the boundary conditions and the related Green function. But, as is well known, the actual implementation of the source function method in applications is often intricate owing to the difficulty to obtain a closed-form analytical expression for the Green function. This is notably the case for the BVPs for Poisson equation in rectangular domains for which series-only representations of Green functions are used [4], although it is possible in principle to circumvent the difficulty by converting known closed-form results obtained for half-plane or circular domain into the rectangle via conformal transformations. Thus, using a conformal Schwarz-Christoffel transformation mapping the half-plane into the rectangle, one can easily find the closed-form solution of homogeneous Dirichlet problem in terms of Jacobi elliptic functions (see for instance

C 2014 Mamode; licensee Springer. This is an Open Access article distributed under the terms of the Creative Commons Attribution License (http://creativecommons.org/licenses/by/4.0), which permits unrestricted use, distribution, and reproduction in any medium, provided the original work is properly credited. 
$[1,2,5])$, but to date a large number of other BVPs for rectangular Poisson equation still have no analytical closed-form solutions.

We show in the present paper that the solutions of these BVPs amount to solve the Poisson equation on flat tori in the distributional sense i.e. in the space of doubly periodic Schwartz distributions on the Euclidean plane $\mathbb{E}^{2}=\mathbb{R}^{2}$ or $\mathbb{C}[6,7]$. For this, the fundamental solution of the Laplacian is first obtained in Section 2 in terms of elliptic functions using Eisenstein's approach via infinite series over rectangular lattices in $\mathbb{C}$. Secondly, using the method of images for instance, the analytical closed-form representation of Green functions for most BVPs stated for planar Poisson equation in the rectangle may easily be derived as shown in Section 3.

\section{Fundamental solution and concept of horizon}

Most BVPs for rectangular Poisson equation may reduce to solve in a distributional sense an equation of the form $\Delta V=F$ on $\mathbb{E}^{2}$, where the boundary conditions act as source terms and appear in the RHS of the equation. In particular, the equation

$$
\Delta G=\frac{\partial^{2} G}{\partial x^{2}}+\frac{\partial^{2} G}{\partial y^{2}}=\delta(x, y), \quad(x, y) \in \mathbb{R}^{2},
$$

where $\delta(x, y)$ is the Dirac distribution at origin, defines the so-called fundamental solution for the Laplacian on the plane. But, as is well known, the solution of (1) is not unique since given the particular solution $\log \left(x^{2}+y^{2}\right) / 4 \pi$, all solutions of (1) must be written of the form

$$
G(x, y)=\frac{1}{4 \pi} \log \left(x^{2}+y^{2}\right)+h(x, y),
$$

where $h$ is an arbitrary harmonic function in $\mathbb{R}^{2}$ i.e. a solution of $\Delta h=0$. Nevertheless, the fundamental solution is that for which it is usually set $h=$ constant and this may be justified in a heuristic manner as follows. Equation (1) may be seen as establishing a causal connection between a point source located at the origin, and the change produced at point $(x, y)$ to a certain property $G$ of the free space. In that sense, in the absence of source, this spatial property governed by (1) does not change and has to remain uniform: $\Delta h=0$ thus must necessarily imply $h=$ constant. In addition, since the fundamental solution may also reflect some geometrical properties of the space like isotropy, it must possess circular symmetry i.e. must depend only on one variable $r=\sqrt{x^{2}+y^{2}}$ and it is easy to show that the only harmonic functions with circular symmetry are again constant functions.

Thus, all these reasons lead us to define the fundamental solution of Laplace equation on $\mathbb{E}^{2}$ as follows:

$$
G(x, y)=\frac{1}{4 \pi} \log \left(\frac{x^{2}+y^{2}}{r_{0}^{2}}\right)
$$

where $r_{0}$ is an arbitrary constant length we shall call in the sequel horizon. It is common, not to say always, the case, to set $a$ priori for convenience $r_{0}=1$. But, in the light of our concern, such a simplification has two inconveniences: (i) it overshadows the scale invariance property of solution of (1) by the change of coordinates $(x, y)$ into $(\varepsilon x, \varepsilon y)$ for any $\varepsilon>0$ and the consequences for existence of the solution of BVPs (see below); (ii) the concept of 
horizon (which may be chosen as far as wanted) restores the property that $G$ tends to 0 as $\left(x^{2}+y^{2}\right)^{1 / 2} \rightarrow r_{0}$ which is, here at finite distance, the property retained for the fundamental solution of Laplace equation in $(n>2)$-dimensional space for which the horizon is located at infinity.

In this cause-and-effect relationship, the distribution solution $V \in \mathcal{D}^{\prime}\left(\mathbb{R}^{2}\right)$ of BVPs may be readily obtained by 'division' i.e. may be given by the double convolution product,

$$
V=G \stackrel{x}{*} * F=\frac{1}{4 \pi} \log \left(\frac{x^{2}+y^{2}}{r_{0}^{2}}\right) \stackrel{x}{*} * F .
$$

Thus, by a such algebraic approach, the existence of the solution is simply related to the existence of the convolution product in $\mathcal{D}^{\prime}\left(\mathbb{R}^{2}\right)$ hence the causal solution is derived up to a constant provided that

$$
\int_{-\infty}^{+\infty} d \xi \int_{-\infty}^{+\infty} d \eta F(\xi, \eta)<+\infty
$$

We focus our attention in the rest of this note to the case where the source term $F$ is doubly periodic in the $x, y$-variables. We shall see indeed that the BVPs for the rectangle, not surprisingly, are relevant to that case related to the tessellation of the Euclidean plane $\mathbb{E}^{2}$ with an infinite number of identical rectangular tiles in both $x, y$-directions.

Assume that $F$ is of period $2 a$ and $2 b$ in the $x$ - and $y$-variable, respectively. It is thus always possible to define the distribution $F_{0}$ on the rectangle $\{(x, y) \mid-a<x<a,-b<y<b\}$ such that 'by gluing together the pieces' [7]

$$
F(x, y)=\sum_{n=-\infty}^{+\infty} \sum_{m=-\infty}^{+\infty} \delta(x-2 n a) \delta(y-2 m b) \stackrel{x}{*} * F_{0}(x, y)
$$

The distribution $F_{0}$ is thereby biunivocally associated to $F \in \mathcal{D}^{\prime}\left(\mathbb{R}^{2}\right)$ and it is a distribution on the flat torus $\Omega_{a, b}=\mathbb{E}^{2} / \Lambda_{a, b}$, quotient of the Euclidean plane by the lattice $\Lambda_{a, b}=2 a \mathbb{Z}+$ $2 i b \mathbb{Z}$. In order that the condition (3) holds, it is here necessary to prescribe the following compatibility condition:

$$
\int_{-a}^{a} d \xi \int_{-b}^{b} d \eta F_{0}(\xi, \eta)=0,
$$

so that the solution of the Poisson equation $\Delta V=F$, initially posed on $\mathbb{E}^{2}$, is the (doubly periodic) distribution on $\Omega_{a, b}$ formally expressed as the convolution product on the torus,

$$
V=\Psi_{a, b}(x, y) \stackrel{x}{*} \stackrel{y}{*} F_{0}(x, y),
$$

where

$$
\begin{aligned}
\Psi_{a, b}(x, y) & =\sum_{n=-\infty}^{+\infty} \sum_{m=-\infty}^{+\infty} \delta(x-2 n a) \delta(y-2 m b) \begin{array}{l}
x^{y} \\
* * G
\end{array} \\
& =\frac{1}{4 \pi} \sum_{n=-\infty}^{+\infty} \sum_{m=-\infty}^{+\infty} \log \left\{\frac{(x-2 n a)^{2}+(y-2 m b)^{2}}{r_{0}^{2}}\right\} \\
& =\frac{1}{4 \pi} \log \left\{\prod_{n=-\infty}^{+\infty} \prod_{m=-\infty}^{+\infty} \frac{(x-2 n a)^{2}+(y-2 m b)^{2}}{r_{0}^{2}}\right\},
\end{aligned}
$$


provided that the previous series is convergent, which is unfortunately not the case for any fixed $r_{0}$.

To overcome this difficulty, let us notice that the solution $V$ should be unchanged owing to the compatibility condition (4), if the term $r_{0}^{2}$ is replaced by

$$
r_{n m}^{2}=4 n^{2} a^{2}+4 m^{2} b^{2} \quad \text { for }|n|+|m| \neq 0
$$

(the nonzero term $r_{00}$ is not specified, as its value is not essential due to the compatibility condition (4)). In other words, the horizon of $(n, m)$ th term of the series is not at all taken constant but is assumed to correspond to the distance from the center of rectangular tile $\{(2 n-1) a<x<(2 n+1) a,(2 m-1) b<y<(2 m+1) b\}$ to the origin $v i z$. the center of fundamental rectangle. In this way, it follows that the double infinite product (6) may be seen as the modulus square of

$$
\phi(z)=\frac{z}{r_{00}} \prod_{n=-\infty}^{+\infty} \prod_{m=-\infty}^{+\infty}\left(1+\frac{z}{2 n a+i 2 m b}\right), \quad z=x+i y,
$$

where the dash, as is customary, denotes omission of the term in $n=m=0$. Nevertheless, the product (8) is always not absolutely convergent and has not the rearrangement property i.e. its limit $\phi(z)$ (if it exists) is dependent of the order of the factors. Using thus the Eisenstein convention [8] which groups together the factors of opposite rank according to the following calculation process:

$$
\lim _{M \rightarrow+\infty} \prod_{m=-M}^{+M} \lim _{N \rightarrow+\infty} \prod_{n=-N}^{+N}
$$

(the order of limits is imposed; see [8]), the specific result denoted $\phi_{e}(z)$ becomes an entire function having zeros only at points of the lattice $\Lambda_{a, b}$ but is not doubly periodic in virtue of Liouville's theorem despite appearances. Indeed, using the Eisenstein convention, (8) shows at once that $\phi_{e}(z+2 a)=-\phi_{e}(z)$ and so $\phi_{e}$ has period $4 a$, while under translation along the imaginary axis,

$$
\phi_{e}(z+2 i b)=-e^{\pi b / a} e^{-i \pi z / a} \phi_{e}(z) .
$$

See [8] for a proof. Hence, following Eisenstein's approach to elliptic functions via infinite series over lattices in $\mathbb{C}[8]$, we may write

$$
\phi_{e}(z) \propto \vartheta_{1}\left(\frac{\pi z}{2 a} \mid i \frac{b}{a}\right)
$$

where $\vartheta_{1}$ denotes the first Jacobi theta function [9]. At this point, it is no longer difficult to show that the convolution kernel $\Psi_{a, b}$, up to a constant, is thus explicitly given by

$$
\Psi_{a, b}(x, y)=\frac{1}{2 \pi} \log \left\{e^{-\pi y^{2} / 4 a b}\left|\vartheta_{1}\left(\frac{\pi}{2 a}(x+i y) \mid i \frac{b}{a}\right)\right|\right\} .
$$


Indeed, firstly $\Psi_{a, b} \in \mathcal{D}^{\prime}\left(\Omega_{a, b}\right)$ and the double periodicity is recovered due to the exponential term since by (9),

$$
\begin{aligned}
\Psi_{a, b}(x, y+2 b) & =\frac{1}{2 \pi} \log \left\{e^{-\pi(y+2 b)^{2} / 4 a b}\left|\phi_{e}(z+2 i b)\right|\right\} \\
& =\frac{1}{2 \pi} \log \left\{e^{-\pi y^{2} / 4 a b}\left|\phi_{e}(z)\right|\right\}=\Psi_{a, b}(x, y) .
\end{aligned}
$$

Secondly, it can be proved that

$$
\Delta \Psi_{a, b}=\delta(x) \delta(y)-\frac{1}{4 a b}
$$

on the flat torus $\Omega_{a, b}$ i.e. $\Psi_{a, b}$ is the fundamental solution (Green function) for the Laplacian on $\Omega_{a, b}$. Indeed, $\phi_{e}(z)$ is zero at $z=0$ so that $\Delta \Psi_{a, b}$ has a singularity at the center of the torus and,

$$
\Delta \Psi_{a, b}+\frac{1}{4 a b}=4 \Re \frac{\partial^{2}}{\partial z \partial \bar{z}} \log \phi_{e}(z)=0 \quad \text { for } z \neq 0
$$

(here and in the sequel, the principal branch of the logarithm is considered). In addition, applying the Green theorem on a fundamental domain of the torus,

$$
\begin{aligned}
\iint_{\Omega_{a, b}}\left(\Delta \Psi_{a, b}+\frac{1}{4 a b}\right) d x d y & =\frac{1}{2 \pi} \iint_{\Omega_{a, b}} \Delta \log \left|\phi_{e}(z)\right| d x d y \\
& =\frac{1}{2 \pi} \Im \oint_{\partial \Omega_{a, b}} \frac{\partial}{\partial z} \log \phi_{e}(z) d z .
\end{aligned}
$$

Since

$$
\frac{\partial}{\partial z} \log \phi_{e}(z)=\frac{\pi}{2 a} \frac{d}{d w} \log \vartheta_{1}\left(w \mid i \frac{b}{a}\right)=\zeta(z)-\frac{\zeta(a)}{a} z \quad \text { with } w=\frac{\pi z}{2 a},
$$

$\zeta(z)$ being the $\zeta$-Weierstrass elliptic function, which is meromorphic, having a simple pole at the origin (and no others on the integration domain) with residue 1 [9], it follows from the residue theorem that

$$
\iint_{\Omega_{a, b}}\left(\Delta \Psi_{a, b}+\frac{1}{4 a b}\right) d x d y=\frac{1}{2 \pi} \Im \oint_{\partial \Omega_{a, b}}\left(\zeta(z)-\frac{\zeta(a)}{a} z\right) d z=1
$$

This completes the proof. A surface plot of the fundamental solution $\Psi_{a, b}$ is given in Figure 1 for $a=b=3$.

Remarks (i) Let us notice that interchanging the roles of variables $x$ and $y$ in what precedes yields an equivalent formulation of the fundamental solution,

$$
\Psi_{a, b}(x, y)=\frac{1}{2 \pi} \log \left\{e^{-\pi x^{2} / 4 a b}\left|\vartheta_{1}\left(\frac{\pi}{2 b}(y+i x) \mid i \frac{a}{b}\right)\right|\right\},
$$

or in a more symmetrical form,

$$
\Psi_{a, b}(x, y)=\frac{1}{4 \pi} \log \left\{e^{-\pi\left(x^{2}+y^{2}\right) / 4 a b}\left|\vartheta_{1}\left(\frac{\pi}{2 a}(x+i y) \mid i \frac{b}{a}\right) \vartheta_{1}\left(\frac{\pi}{2 b}(y+i x) \mid i \frac{a}{b}\right)\right|\right\} .
$$




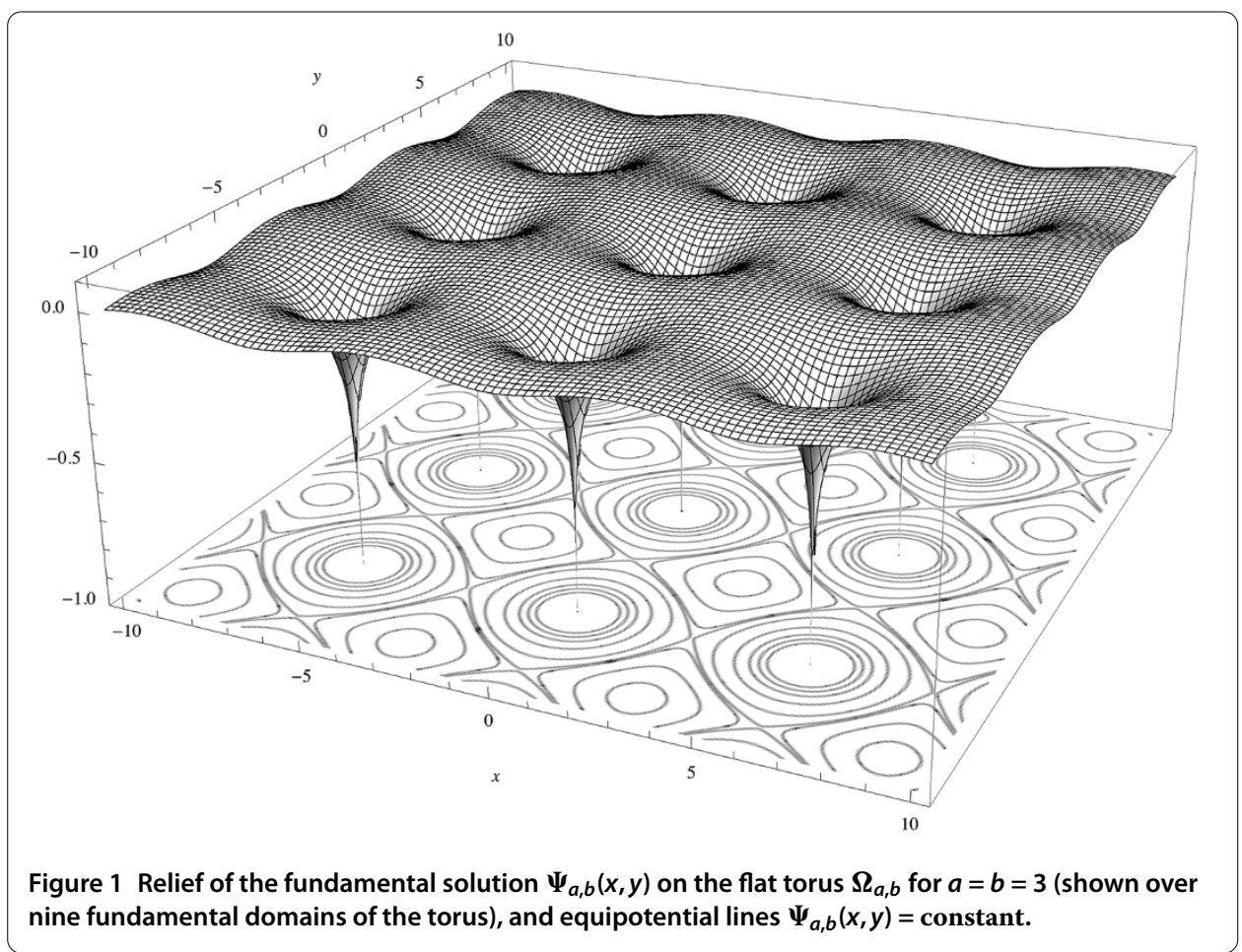

(ii) Since from (11),

$$
\begin{aligned}
\Delta^{2}\left(\Psi_{a, b} * \stackrel{x}{*} * \Psi_{a, b}\right) & =\Delta \Psi_{a, b} \stackrel{x}{*} * \Delta \Psi_{a, b} \\
& =\left(\delta(x) \delta(y)-\frac{1}{4 a b}\right) * * \begin{array}{l}
* y \\
* *
\end{array}\left(\delta(x) \delta(y)-\frac{1}{4 a b}\right) \\
& =\delta(x) \delta(y)-\frac{1}{4 a b},
\end{aligned}
$$

it follows by induction that, for any integer $n \geq 1$,

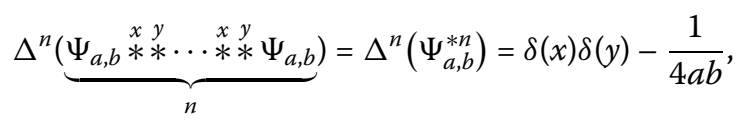

which shows that the convolution power $\Psi_{a, b}^{* n}(x, y)$ is the fundamental solution for the ( $n \geq$ 1)-Laplacian on the flat torus $\Omega_{a, b}$.

\section{Applications}

Having found the fundamental solution for the Laplacian (10), it is straightforward using the image method (by an appropriate periodic distribution of images) to obtain the exact expression of the Green function for most BVPs stated for Poisson equation in the rectangle.

Thus, for the Dirichlet problem, the related Green function $G_{D}\left(x, y ; x_{0}, y_{0}\right)$ i.e. the solution of the equation $\Delta G_{D}=\delta\left(x-x_{0}\right) \delta\left(y-y_{0}\right)$ on the rectangle $\{(x, y) \mid 0<x<a, 0<y<b\}$ with the homogeneous conditions $G_{D}=0$ on the sides, or equivalently the periodic distri- 
bution solution of

$$
\Delta G_{D}=\delta\left(x-x_{0}\right) \delta\left(y-y_{0}\right)-\delta\left(x+x_{0}\right) \delta\left(y-y_{0}\right)-\delta\left(x-x_{0}\right) \delta\left(y+y_{0}\right)+\delta\left(x+x_{0}\right) \delta\left(y+y_{0}\right)
$$

on the flat torus $\Omega_{a, b}$ (note that the compatibility condition (4) is well fulfilled) is simply given by

$$
\begin{aligned}
G_{D}\left(x, y ; x_{0}, y_{0}\right)= & \Psi_{a, b}\left(x-x_{0}, y-y_{0}\right)-\Psi_{a, b}\left(x+x_{0}, y-y_{0}\right) \\
& -\Psi_{a, b}\left(x-x_{0}, y+y_{0}\right)+\Psi_{a, b}\left(x+x_{0}, y+y_{0}\right),
\end{aligned}
$$

i.e. by setting $z=x+i y$ and $z_{0}=x_{0}+i y_{0}$,

$$
G_{D}\left(x, y ; x_{0}, y_{0}\right)=\frac{1}{2 \pi} \Re \log \left\{\frac{\vartheta_{1}\left(\frac{\pi}{2 a}\left(z-z_{0}\right) \mid i \frac{b}{a}\right) \vartheta_{1}\left(\frac{\pi}{2 a}\left(z+z_{0}\right) \mid i \frac{b}{a}\right)}{\vartheta_{1}\left(\frac{\pi}{2 a}\left(z+\bar{z}_{0}\right) \mid i \frac{b}{a}\right) \vartheta_{1}\left(\frac{\pi}{2 a}\left(z-\bar{z}_{0}\right) \mid i \frac{b}{a}\right)}\right\} .
$$

This result is to be compared with that obtained in [5]. Here, it is worth to note that in addition to its simplicity, the complex formulation of the Green function together with the properties of functions of a complex variable and of conformal transformation may provide us with several methods for extending as desired the Dirichlet problem to many other geometries [10].

Similarly, for the Neumann problem, the 'generalized Green function' (also called Neumann function) $G_{N}\left(x, y ; x_{0}, y_{0}\right)$ i.e. the solution of the equation $\Delta G_{N}=\delta\left(x-x_{0}\right) \delta(y-$ $\left.y_{0}\right)-\frac{1}{a b}$ on the rectangle $\{(x, y) \mid 0<x<a, 0<y<b\}$ with the homogeneous conditions $\frac{\partial G_{N}}{\partial n}=0$ on the sides $(\partial / \partial n$ denotes as usual the outward normal derivative; notice that here the compatibility condition (4) is nothing but the Gauss theorem), or equivalently the periodic distribution solution of

$$
\begin{aligned}
\Delta G_{N}= & \delta\left(x-x_{0}\right) \delta\left(y-y_{0}\right)+\delta\left(x+x_{0}\right) \delta\left(y-y_{0}\right)+\delta\left(x-x_{0}\right) \delta\left(y+y_{0}\right) \\
& +\delta\left(x+x_{0}\right) \delta\left(y+y_{0}\right)-\frac{1}{a b}
\end{aligned}
$$

on the flat torus $\Omega_{a, b}$ is thus given by

$$
\begin{aligned}
G_{N}\left(x, y ; x_{0}, y_{0}\right)= & \Psi_{a, b}\left(x-x_{0}, y-y_{0}\right)+\Psi_{a, b}\left(x+x_{0}, y-y_{0}\right) \\
& +\Psi_{a, b}\left(x-x_{0}, y+y_{0}\right)+\Psi_{a, b}\left(x+x_{0}, y+y_{0}\right),
\end{aligned}
$$

i.e.

$$
\begin{aligned}
G_{N}\left(x, y ; x_{0}, y_{0}\right)= & -\frac{y^{2}+y_{0}^{2}}{2 a b}+\frac{1}{2 \pi} \Re \log \left\{\vartheta_{1}\left(\frac{\pi}{2 a}\left(z-z_{0}\right) \mid i \frac{b}{a}\right) \vartheta_{1}\left(\frac{\pi}{2 a}\left(z+z_{0}\right) \mid i \frac{b}{a}\right)\right. \\
& \left.\times \vartheta_{1}\left(\frac{\pi}{2 a}\left(z+\bar{z}_{0}\right) \mid i \frac{b}{a}\right) \vartheta_{1}\left(\frac{\pi}{2 a}\left(z-\bar{z}_{0}\right) \mid i \frac{b}{a}\right)\right\} .
\end{aligned}
$$

To illustrate the practical value of such an analytical result, consider the Poisson equation $\Delta u=f(x, y)$ on the unit square $\{0<x, y<1\}$ subject to homogeneous Neumann boundary conditions and where

$$
f(x, y)=\delta(x-\varepsilon, y-\varepsilon)-\delta(x-1+\varepsilon, y-1+\varepsilon), \quad 0<\varepsilon<1,
$$




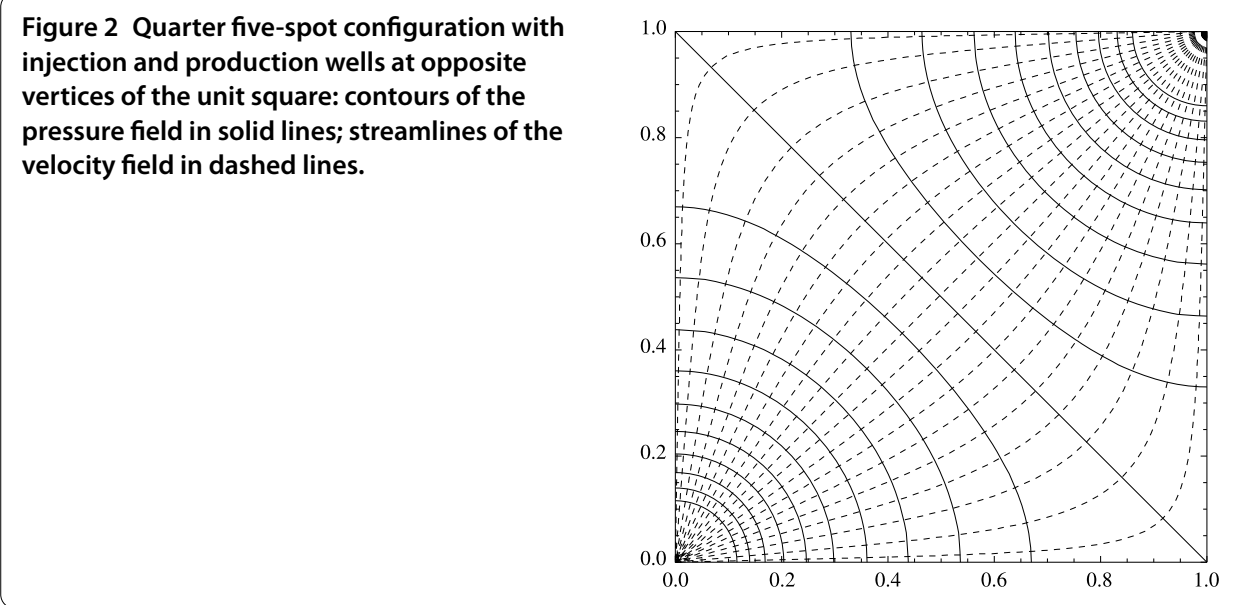

models two unit points sources of opposite strength and located on a diagonal of the square. The solution is at once given up to a constant by

$$
\begin{aligned}
u(x, y) & =\int_{0}^{1} d \xi \int_{0}^{1} d \eta G_{N}(x, y ; \xi, \eta) f(\xi, \eta) \\
& =G_{N}(x, y ; \varepsilon, \varepsilon)-G_{N}(x, y ; 1-\varepsilon, 1-\varepsilon)
\end{aligned}
$$

and, taking the limit $\varepsilon \rightarrow 0$, the result obtained after simplification,

$$
u(x, y)=\frac{2}{\pi} \Re \log \left\{\frac{\vartheta_{1}\left(\frac{\pi}{2} z \mid i\right)}{\vartheta_{3}\left(\frac{\pi}{2} z \mid i\right)}\right\}, \quad z=x+i y
$$

may be considered as the exact analytical solution of a standard porous media problem known as the homogeneous quarter five-spot problem which is a popular test case scenario in oil reservoir simulation [11]. Figure 2 shows the contours of the pressure field (15) and the streamlines of the velocity field defined by the curves

$$
\Im \log \left\{\frac{\vartheta_{1}\left(\frac{\pi}{2} z \mid i\right)}{\vartheta_{3}\left(\frac{\pi}{2} z \mid i\right)}\right\}=\text { constant, }
$$

which agree well with numerical simulations [11].

\section{Perspectives and conclusions}

The search of analytical closed-form solutions for BVPs for planar Poisson equation - but this is also true for many other problems of mathematical physics - are nowadays little tackled for the benefit of numerical computational methods while the existence of a compact analytical closed-form solution for such problems is always a great advance for a better understanding of underlying physics. The present work has developed a simple method for analytically solving most BVPs stated for the planar Poisson equation in a rectangular domain for which, to our knowledge, only series representation of solutions were available. This construction, based on the Green function method, amounts to solve the Poisson equation on flat tori in the distributional sense having found the fundamental solution of the Laplacian in terms of elliptic functions. When it is possible, the complex formulation of obtained results will allow one to extend - via conformal transformations - the 
number of problems posed on regions of more complicated shape and with a mixed setting of different kinds of boundary conditions. Although it was not the primary object of this note, having obtained the fundamental solution of $n$-Laplacian on the flat torus will also be of great interest for explicitly solving BVPs for two-dimensional $n$-harmonic equation in rectangles as, for instance, the biharmonic equation and the linear clamped plate boundary value problem in mechanics.

\section{Competing interests}

The author declares that he has no competing interests.

\section{Acknowledgements}

The author would like to thank the referees for their valuable remarks and suggestions.

Received: 11 July 2014 Accepted: 16 September 2014 Published online: 01 October 2014

\section{References}

1. Morse, PM, Feshbach, H: Methods of Theoretical Physics, vol. 1. McGraw-Hill, New York (1953)

2. Morse, PM, Feshbach, H: Methods of Theoretical Physics, vol. 2. McGraw-Hill, New York (1953)

3. Tikhonov, AN, Samarskii, AA: Equations of Mathematical Physics. Dover, New York (1990)

4. Melnikov, YA, Melnikov, MY: Computability of series representations for Green's functions in a rectangle. Eng. Anal. Bound. Elem. 30, 774-780 (2006)

5. Shou, Q, Jiang, Q, Guo, Q: The closed-form solution for the 2D Poisson equation with a rectangular boundary. J. Phys. A, Math. Theor. 42, 205202 (2009)

6. Gel'fand, IM, Shilov, GE: Generalized Functions, vol. I. Academic Press, New York (1964)

7. Schwartz, L: Mathematics for the Physical Sciences. Addison-Wesley, Reading (1966)

8. Walker, PL: Elliptic Functions: A Constructive Approach. Wiley, New York (1996)

9. Gradshteyn, IS, Ryzhik, IM: Table of Integrals, Series and Products. Academic Press, New York (2000)

10. Driscoll, TA, Trefethen, LN: Schwarz-Christoffel Mapping. Cambridge Monographs on Applied and Computational Mathematics, vol. 8. Cambridge University Press, Cambridge (2002)

11. Chen, CY, Meiburg, E: Miscible porous media displacements in the quarter five-spot configuration. Part 1. The homogeneous case. J. Fluid Mech. 371, 233-268 (1998)

doi:10.1186/s13661-014-0221-4

Cite this article as: Mamode: Fundamental solution of the Laplacian on flat tori and boundary value problems for the planar Poisson equation in rectangles. Boundary Value Problems 2014 2014:221.

\section{Submit your manuscript to a SpringerOpen ${ }^{\circ}$ journal and benefit from:}

- Convenient online submission

Rigorous peer review

- Immediate publication on acceptance

- Open access: articles freely available online

- High visibility within the field

- Retaining the copyright to your article 